https://doi.org/10.15407/ujpe63.3.196

V.E. KUZMICHEV, V.V. KUZMICHEV

Bogolyubov Institute for Theoretical Physics, Nat. Acad. of Sci. of Ukraine

(14-b, Metrolohichna Str., Kyiv03143, Ukraine; e-mail: vkuzmichev@bitp.kiev.ua)

\title{
QUANTUM DYNAMICS OF THE EARLY UNIVERSE
}

\begin{abstract}
Quantum gravity may shed light on the prehistory of the universe. Quantum corrections to gravity affect the dynamics of the expansion of the universe. Their influence is studied on the example of the exactly solvable quantum model. The corrections to the energy density and pressure lead to the emergence of an additional attraction (like dark matter) or repulsion (like dark energy) in the quantum system of the gravitating matter and radiation. The model explains the accelerating expansion (inflation) in the early universe (the domain of comparatively small values of quantum numbers) and a later transition from the decelerating expansion to the accelerating one of the universe (the domain of very large values of quantum numbers) from a single approach. The generation of primordial fluctuations of the energy density at the expense of the change of a sign of the quantum correction to the pressure is discussed.

Keywords: quantum gravity, quantum geometrodynamics, cosmology.
\end{abstract}

\section{Introduction}

Quantum gravity may shed light on the prehistory of the universe before the big-bang scenario takes over. Numerous different models were proposed to reconcile the classical theory of gravity, based on general relativity, with current astrophysical data [1-3]. The transition from the classical model of the universe to its quantum analog adds a new element to the theory in the form of quantum corrections to the energy density and pressure. The method of constraint system quantization can be taken as a basis of the quantum theory of gravity suitable for the investigation of cosmological and other quantum gravitational systems. In this article, we use quantum geometrodynamics in order to study an influence of quantum corrections on the dynamics of a quantum gravitational system (universe). For this purpose, it is reasonable to investigate this problem within an exactly solvable cosmological model.

The canonical approach to the quantization, successful in constructing the nonrelativistic quantum mechanics and quantum field theories in the flat spacetime, encounters well-known difficulties, when applied to gravity, such as the understanding of the time evolution, the divergence of the norm of the state vectors, the measurement problem, and others. A model with a finite number of degrees of freedom

(c) V.E. KUZMICHEV, V.V. KUZMICHEV, 2018 may provide a reasonable framework for addressing the problems of quantum gravity. The homogeneous minisuperspace models have been proven to be successful - consistent with observations and having predictive power - in classical cosmology. This appears explicable, in view of the fact that the Universe can, in the first approximation, be considered as being homogeneous, and gives rise to the hope for that homogeneous models could be useful in quantum cosmology as well.

In Section 2, a short introduction to general relativity as a theory with constraints is made on the example of the minisuperspace model. Section 3 is devoted to the formulation of a version of quantum theory of gravitational systems in the maximally symmetric space. In Sections 4 and 5, quantum analogs of the Hamilton-Jacobi equation and the EinsteinFriedmann equation are obtained. The goal of Section 6 is to demonstrate on a concrete example how quantum effects manifest themselves in the early universe, by determining the behavior of the cosmological parameters such as the energy density, pressure, and deceleration parameter under the expansion of the universe. In Section 7, the results are discussed.

\section{Constraint Equations}

\section{for the Maximally Symmetric Geometry}

We will consider an isotropic cosmological model described by the Friedmann-Robertson-Walker metric.

ISSN 2071-0194. Ukr. J. Phys. 2018. Vol. 63, No. 3 
In the case of the maximally symmetric geometry, the geometric properties of the system are determined by a single variable, namely the cosmic scale factor $a$. The matter sector is taken in the form of a uniform scalar field $\phi$ (a surrogate of physical fields) and a perfect fluid which defines the material reference frame enabling one to recognize the instants of time. The Hamiltonian of the system has the form [4-8]

$H=\frac{N}{2}\left\{-\pi_{a}^{2}-a^{2}+a^{4}\left[\rho_{\phi}+\rho_{\gamma}\right]\right\}+$

$+c_{1}\left\{\pi_{\Theta}-\frac{1}{2} a^{3} \rho_{0} s\right\}+c_{2}\left\{\pi_{\tilde{\lambda}}+\frac{1}{2} a^{3} \rho_{0}\right\}$,

where $\pi_{a}, \pi_{\Theta}$, and $\pi_{\tilde{\lambda}}$ are the momenta canonically conjugate with the variables $a, \Theta$ (the thermasy), and $\tilde{\lambda}$ (the potential for the specific free energy taken with an inverse sign), $\rho_{\phi}$ is the energy density of matter represented by a scalar field $\phi, \rho_{\gamma}$ is the energy density of a perfect fluid, which is a function of the density of the rest mass $\rho_{0}$ and the specific entropy $s[9] ; N, c_{1}$, and $c_{2}$ play the role of the Lagrange multipliers.

Here and below, we use the Planck system of units. The length $l_{\mathrm{P}}=\sqrt{2 G \hbar /\left(3 \pi c^{3}\right)}$ is taken as a unit of length, and the $\rho_{\mathrm{P}}=3 c^{4} /\left(8 \pi G l_{\mathrm{P}}^{2}\right)$ is used as a unit of energy density and pressure, where $G$ is Newton's gravitational constant. The proper time $\tau$ is measured in units of length.

Hamiltonian (1) is a linear combination of constraints and thus weakly vanishes, $H \approx 0$. The variations of the Hamiltonian with respect to $N, c_{1}$, and $c_{2}$ give three constraint equations,

$-\pi_{a}^{2}-a^{2}+a^{4}\left[\rho_{\phi}+\rho_{\gamma}\right] \approx 0$,

$\pi_{\Theta}-\frac{1}{2} a^{3} \rho_{0} s \approx 0, \quad \pi_{\tilde{\lambda}}+\frac{1}{2} a^{3} \rho_{0} \approx 0$.

From the conservation of these constraints in time, it follows that the number of particles of a perfect fluid in the proper volume $\frac{1}{2} a^{3}$ (or $2 \pi^{2} a^{3}$, if $a$ is taken in units of length) and the specific entropy conserve: $\frac{1}{2} a^{3} \rho_{0}=$ const, $s=$ const. In view of these conservation laws and the vanishing of the momenta conjugate with the variables $\rho_{0}$ and $s$, one can discard the degrees of freedom corresponding to these variables and convert the second-class constraints into firstclass constraints [4-6], in accordance with Dirac's proposal.

\section{Quantization Scheme}

In quantum theory, first-class constraint equations become constraints on the state vector and, in this way, define the space of physical states [10]. It is convenient to choose the perfect fluid with the density $\rho_{\gamma}$ in the form of relativistic matter (radiation). Then one can put $a^{4} \rho_{\gamma} \equiv E=$ const. Passing from the classical variables in the constraint equations (2) to corresponding operators, we obtain the quantum constraint equations, which vanish when applied to the state vector $\langle a, \phi \mid \Psi(T)\rangle$, where $T$ is the "arc-parameter measure of time" connected with the proper time $\tau$ by the differential equation $d \tau=a d T$ $[7,8]$. The solution of these equations gives the evolution of $|\Psi(T)\rangle$ in the time parameter $T$ in the form

$|\Psi(T)\rangle=e^{i \frac{2}{3} E\left(T-T_{0}\right)}\left|\Psi\left(T_{0}\right)\right\rangle$,

where $T_{0}$ is an arbitrary constant taken as a time reference point. The vector $\left|\Psi\left(T_{0}\right)\right\rangle \equiv|\psi\rangle$ satisfies the equation

$\left(-\partial_{a}^{2}+a^{2}-2 a \hat{H}_{\phi}\right)|\psi\rangle=E|\psi\rangle$.

The operators

$\hat{H}_{\phi}=\frac{1}{2} a^{3} \hat{\rho}_{\phi}, \quad \hat{L}_{\phi}=\frac{1}{2} a^{3} \hat{p}_{\phi}$

are the Hamiltonian and the Lagrangian of the scalar field $\phi$, where

$\hat{\rho}_{\phi}=-\frac{2}{a^{6}} \partial_{\phi}^{2}+V(\phi), \quad \hat{p}_{\phi}=-\frac{2}{a^{6}} \partial_{\phi}^{2}-V(\phi)$,

are the operators of energy density and pressure, respectively, and $V(\phi)$ is the potential term.

In the case $E=0$, Eq. (4) reduces to the WheelerDeWitt equation of a 'minisuperspace model' of the universe filled with a uniform scalar field.

The Hamiltonian $\hat{H}_{\phi}$ can be diagonalized by introducing the complete set of orthonormalized functions $\left\langle\chi \mid u_{k}\right\rangle$ of a quantum scalar field in the representation of the generalized variable $\chi=\chi\left(\frac{1}{2} a^{3}, \phi\right)$. The explicit form of $\chi$ is determined by the form of the potential $V(\phi)$ [7]. For example, in the model $V(\phi)=$ $=\lambda_{\alpha} \phi^{\alpha}$, where $\lambda_{\alpha}$ is the coupling constant and $\alpha \geq 0$, we have

$\chi=\left(2 \lambda_{\alpha}\right)^{\frac{1}{2+\alpha}}\left(\frac{a^{3}}{2}\right)^{\frac{2}{2+\alpha}} \phi$. 
After averaging over the quantum states $\left|u_{k}\right\rangle$, the scalar field turns into matter characterized by the energy density $\rho_{m}$ and pressure $p_{m}$,

$\rho_{m}=\left\langle u_{k}\left|\hat{\rho}_{\phi}\right| u_{k}\right\rangle, \quad p_{m}=\left\langle u_{k}\left|\hat{p}_{\phi}\right| u_{k}\right\rangle$.

The expectation value of the Hamiltonian is

$\left\langle u_{k}\left|\hat{H}_{\phi}\right| u_{k^{\prime}}\right\rangle=M_{k}(a) \delta_{k k^{\prime}}$,

where the index of the state $k$ can take both discrete and continuous values, $M_{k}(a)=\frac{1}{2} a^{3} \rho_{m}$ is the proper energy of matter in the volume $\frac{1}{2} a^{3}$ with the energy density $\rho_{m}$.

Calculating the expectation values (7), we obtain

$\rho_{m}=\frac{2 M_{k}(a)}{a^{3}}, \quad p_{m}=w_{m} \rho_{m}$,

where the equation-of-state parameter

$w_{m}=-\frac{1}{3} \frac{d \ln M_{k}(a)}{d \ln a}$.

In the general case, the proper energy $M_{k}(a)$ depends on $a$. It describes a classical source (as a massenergy) of the gravitational field.

In the specific case, in the model $V(\phi)=\lambda_{\alpha} \phi^{\alpha}$, matter reduces to a barotropic fluid with the parameter $w_{m}=\frac{\alpha-2}{\alpha+2}$, which does not depend on $a$. For $\alpha=0$, the barotropic fluid takes the form of the vacuum of the scalar field in the $k$ th state. The value $\alpha=1$ corresponds to the strings. Matter in the form of dust is reproduced by $\alpha=2$, whereas $\alpha=4$ leads to the relativistic matter and so on. The so-called stiff Zel'dovich matter is obtained in the limiting case $\alpha=\infty$.

\section{Quantum Hamilton-Jacobi Equation}

Using Eq. (8), one can integrate Eq. (4) with respect to the matter field variable. Let us express the vector $|\psi\rangle$ as the expansion in the complete set of states $\left|u_{k}\right\rangle$,

$|\psi\rangle=\sum_{k}\left|u_{k}\right\rangle\left\langle u_{k} \mid \psi\right\rangle$.

Then Eq. (4) yields the equation for the function $\left\langle a \mid f_{k}\right\rangle \equiv\left\langle u_{k} \mid \psi\right\rangle$,

$\left(-\partial_{a}^{2}+a^{2}-2 a M_{k}(a)\right)\left|f_{k}\right\rangle=E\left|f_{k}\right\rangle$.
This equation is an eigenvalue equation. Its solution $\left|f_{k}\right\rangle$ is an eigenfunction corresponding to the eigenvalue $E$. The function $\left|f_{k}\right\rangle$ describes the geometric properties of the quantum universe filled with matter, whose mass-energy is $M_{k}(a)$.

In order to turn to the classical observables, we shall look for the solution of Eq. (12) in the form

$\left\langle a \mid f_{k}\right\rangle=\frac{C_{k}}{\sqrt{\partial_{a} S_{k}(a)}} e^{i S_{k}(a)}$,

where the phase $S_{k}$ can, generally speaking, be complex and $C_{k}$ is the constant determined by the boundary condition on the function $\left\langle a \mid f_{k}\right\rangle$, e.g. on the asymptotics $a \rightarrow \infty$.

The phase $S_{k}(a)$ satisfies the non-linear equation

$\left(\partial_{a} S_{k}\right)^{2}+a^{2}-2 a M_{k}(a)-E=\frac{3}{4}\left(\frac{\partial_{a}^{2} S_{k}}{\partial_{a} S_{k}}\right)^{2}-\frac{1}{2} \frac{\partial_{a}^{3} S_{k}}{\partial_{a} S_{k}}$.

This equation is exact. It is equivalent to Eq. (12). In the classical limit, the right-hand side of Eq. (14) vanishes (it is proportional to $l_{\mathrm{P}}^{4}$ in physical units) and this equation turns into the Hamilton-Jacobi equation for the action $\left.S_{k}(a)\right|_{\hbar=0}=S_{k}^{\text {class }}(a)$, and the momentum $\partial_{a} S_{k}^{\text {class }}(a)=-a \dot{a}$, where a dot is used to denote the derivative with respect to the proper time $\tau$. This simple equation is modified, when quantum gravity effects are taken into account.

\section{Quantum Analog of Einstein-Friedmann Equation}

Using Eqs. (11) and (13), from the equation of motion $\left\langle\psi\left|-i \partial_{a}\right| \psi\right\rangle=\left\langle\psi\left|-\frac{d a}{d T}\right| \psi\right\rangle[7,8]$, we get the operator equality

$\partial_{a} S_{k}+\frac{i}{2} \frac{\partial_{a}^{2} S_{k}}{\partial_{a} S_{k}}=-\frac{d a}{d T}$

From this equation, it follows that, generally speaking, the scale factor $a$ can be complex. The possibility of the introduction of a complex metric tensor and its relation to real physical gravitational field were studied in $[8,11,12]$. Taking the common point of view, we shall assume that the physical gravitational field is described by the real part of the metric.

Using Eqs. (13) and (15), Eq. (14) can be rewritten in the form of the Einstein-Friedmann equation

$\left(\frac{\dot{a}}{a}\right)^{2}=\rho_{m}+\rho_{\gamma}+\rho_{q}-\frac{1}{a^{2}}$.

ISSN 2071-0194. Ukr. J. Phys. 2018. Vol. 63, No. 3 
Differentiating Eq. (16) with respect to the proper time $\tau$ and taking the first law of thermodynamics into account, we have

$\frac{\ddot{a}}{a}=-\frac{1}{2}\left[\rho_{m}+\rho_{\gamma}+\rho_{q}+3\left(p_{m}+p_{\gamma}+p_{q}\right)\right]$.

Here, $\rho_{m}$ and $p_{m}$ are the energy density and the pressure of matter with the equation-of-state parameter (10), $\rho_{\gamma}$ and $p_{\gamma}$ are the energy density and the pressure of radiation with the equation of state $p_{\gamma}=\frac{1}{3} \rho_{\gamma}$ $\rho_{q}$ and $p_{q}$ are the quantum corrections to the total energy density and pressure, which have the form

$\rho_{q}=\frac{Q_{k}(a)}{a^{4}} \equiv \frac{2 M_{Q}(a)}{a^{3}}, \quad p_{q}=w_{q} \rho_{q}$,

where $M_{Q}(a)=\frac{1}{2} a^{3} \rho_{q}$ is the proper energy of the quantum source of the gravitational field and the equation-of-state parameter

$w_{q}=\frac{1}{3}\left(1-\frac{d \ln Q_{k}(a)}{d \ln a}\right)$

In Eq. (19), the first term is a correction for relativity, while the second one comes from the quantum dynamics of the system, and it is expressed via the function of the gravitational quantum source

$Q_{k}(a)=i \partial_{a}^{2} S_{k}+\frac{1}{2}\left[\left(\frac{\partial_{a}^{2} S_{k}}{\partial_{a} S_{k}}\right)^{2}-\frac{\partial_{a}^{3} S_{k}}{\partial_{a} S_{k}}\right]$.

Equations (16) and (17) rewritten in physical units demonstrate that the energy density $\rho_{q}$ and the pressure $p_{q}$ contain the terms proportional to $l_{\mathrm{P}}^{2}$ (from the first term on the right-hand side of Eq. (20)) and $l_{\mathrm{P}}^{4}$ (from the term in square brackets).

The function of the gravitational quantum source $Q_{k}$ is a real-valued function, only when the phase can be represented in the form $S_{k}=i S_{k}^{\text {Euclid }}$ with the real-valued Euclidean action. The influence of the gravitational quantum source on the dynamics of the expanding universe depends on the value and the sign of the corresponding energy density and pressure.

If there exists the domain, where the real-valued function $Q_{k}(a)>0$ and $\ln Q_{k}(a)$ depends on $\ln a$, so that $w_{q}$ can be parametrized in the form $w_{q}=-\frac{1}{3} \delta$, where $\delta$ is an arbitrary positive or negative constant, then the quantum corrections can imitate, for example, the contribution from the de Sitter vacuum $(\delta=3)$, domain walls $(\delta=2)$, strings $(\delta=1)$, dust $(\delta=0)$, radiation $(\delta=-1)$, or perfect gas $(\delta=-2)$. In such a model, the quantum source is $Q_{k}(a) \sim a^{\delta+1}$. Identifying the energy density $\rho_{q}>0$ with the energy density of dark energy, one finds that the case $\delta=3$ reproduces the cosmological constant [13], the values $1<\delta<3$ correspond to the quintessence $[14-16]$, whereas the phantom field $[17,18]$ is described by the values $\delta>3$.

However, it is possible that quantum effects will generate the quantum corrections, for which the function $Q_{k}(a)<0$ and the corresponding energy density is negative. This case is not extraordinary. According to quantum field theory, for instance, vacuum fluctuations make a negative contribution to the field energy per unit area (the Casimir effect). As was shown in Ref. [19], the quantum correction $\rho_{q}$ takes a negative value near the initial cosmological singularity.

\section{Exactly Solvable Model}

In this connection, it is appropriate to study an exactly solvable model with the scalar field potential $V(\phi)=\lambda_{2} \phi^{2}$. Then the mass-energy $M_{k}(a)=$ $=\sqrt{2 \lambda_{2}}\left(k+\frac{1}{2}\right) \equiv M=$ const, where $k=0,1,2, \ldots$ is the number of non-interacting identical particles with the mass $\sqrt{2 \lambda_{2}}$ in the state $\left|u_{k}\right\rangle$ of the scalar field.

Equations (12) and (14) have analytical solutions. The eigenvalue $E$ is determined by the condition of quantization $E=2 n+1-M^{2}$, where $n=0,1,2, \ldots$ is the quantum number, which enumerates the discrete states of the universe in the potential well $\zeta^{2} \equiv(a-M)^{2}$. The phase in Eq. (13) is found to be pure imaginary, $S=i S^{\text {Euclid }}$, where

$S^{\text {Euclid }}=\frac{1}{2} \ln \left[2 \int_{0}^{\zeta} d x e^{x^{2}} H_{n}^{-2}(x)\right]+$ const

while the wave function $\langle a \mid f\rangle$ is real-valued,

$\langle a \mid f\rangle=\left(2^{n} n ! \sqrt{\pi}\right)^{-1 / 2} e^{-(a-M)^{2} / 2} H_{n}(a-M)$,

$H_{n}$ is the Hermitian polynomial. Here and below, the indices $k$ and $n$ for $S, f$, and $Q$ are omitted to simplify the notation. Using

$$
\partial_{\zeta} S(\zeta)=i \frac{e^{\zeta^{2}} H_{n}^{-2}(\zeta)}{2 \int_{0}^{\zeta} d x e^{x^{2}} H_{n}^{-2}(x)},
$$




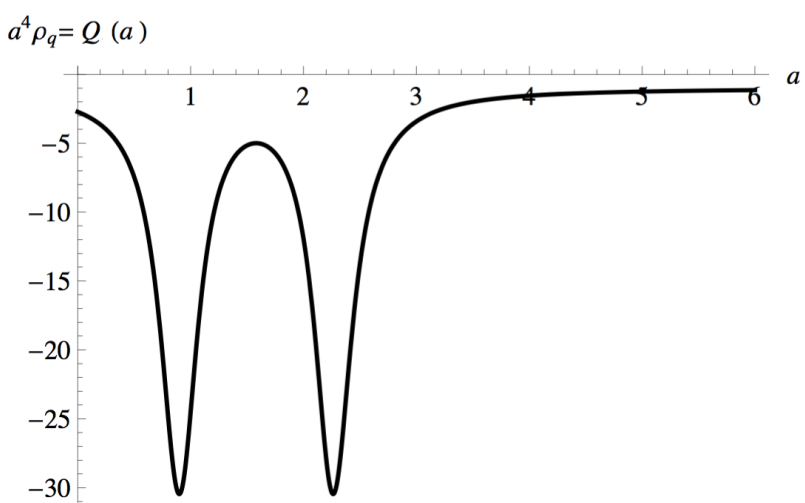

Fig. 1. Quantum corrections $\rho_{q}(18)$ to the total energy density multiplied by $a^{4}$ for the quantum source $Q$ (24) versus the scale factor $a$ for $n=2$ and $M=1.58$

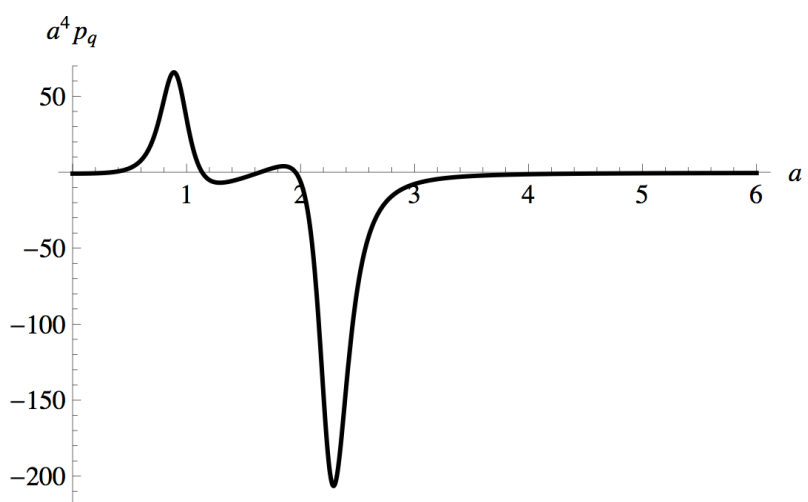

Fig. 2. Quantum corrections $p_{q}(18)$ to the pressure multiplied by $a^{4}$ for the quantum source $Q(24)$ versus the scale factor $a$ for $n=2$

the function of the source $Q(a)$ can be rewritten in

the form
$Q(\zeta)=-(2 n+1)+2 n \frac{H_{n-1}(\zeta) H_{n+1}(\zeta)}{H_{n}^{2}(\zeta)}$.

This function depends on the quantum $n$th state of the universe. The condition $\rho_{\gamma}=\frac{E}{a^{4}} \geq 0$ imposes a restriction on the mass $M$ of the universe, $M \leq \sqrt{2 n+1}$, in accordance with the quantization condition for $E \geq 0$. The observed part of our universe is characterized by the parameters $M \sim 10^{61}\left(\sim 10^{80} \mathrm{GeV}\right)$ and $E \sim 10^{118}\left(\rho_{\gamma} \approx\right.$ $\left.\approx 10^{-10} \mathrm{GeV} / \mathrm{cm}^{3}\right)$. From the viewpoint of the model under consideration, it is in the state with $n \sim$ $\sim 10^{122}$ (up to $\sim 10^{-4}$ ). This estimate practically coincides with the estimate given in Ref. [20]. Therefore, one can put $E=M^{2}$ (for the early universe) and $E=0$ (for the universe with $n \gg 1$ ) for numerical estimations.
From the explicit form of Eq. (24), it follows that the function of the gravitational quantum source $Q(a)$ is negative and oscillates $n$ times in the interval $0 \leq a<\infty$. This function and its first derivative with respect to $a$ diverge at the points $\zeta=\zeta_{i}$, where the Hermitian polynomial $H_{n}(\zeta)$ vanishes, $H_{n}\left(\zeta_{i}\right)=0$. In order to get rid of these divergences, we shall take into account that the scale factor $a$ can be analytically extended to the complex plane (see Eq. (15)). We shift the variable $\zeta$ into the complex domain, $\zeta \rightarrow \zeta+i \varepsilon$, where $\varepsilon^{2} \ll 1$, and substitute

$H_{n}^{2}(\zeta) \rightarrow\left|H_{n}(\zeta+i \varepsilon)\right|^{2}$

in the denominator of Eq. (24). By expanding the square of the polynomial in a small parameter $\varepsilon$ and keeping the first non-vanishing term, we obtain

$\left|H_{n}(\zeta+i \varepsilon)\right|^{2}=H_{n}^{2}(\zeta)+\varepsilon^{2}(2 n)^{2} H_{n-1}^{2}(\zeta)$.

It turns out that the simple regularization

$H_{n}^{2}(\zeta) \rightarrow H_{n}^{2}(\zeta)+\epsilon_{n}$

where $\epsilon_{n}$ is some phenomenological parameter chosen so that the positions and magnitudes of maxima of the function $Q(a)$ do not change, leads to the qualitatively similar results as a shift into the complex domain, thus proving the model-independence of the method.

In Fig. 1 for illustrative purposes, we show the quantum correction $\rho_{q}$ as a function of $a$ with the specific values $n=2, M=1.58$, and $E=M^{2}$, which correspond to the early universe, and $\varepsilon^{2}=0.04$. The main features of the behavior of $\rho_{q}$ remain intact up to the values $n \gg 1$ and $E \ll M^{2}$. They do not depend on the value $\varepsilon^{2}$, which determines only the depths of the minima and does not change the general picture. The quantum correction $\rho_{q}$ is multiplied by $a^{4}$ in order to exclude the divergence at the point of the initial cosmological singularity $a=0$ from the consideration. This quantum correction to the total energy density in Eqs. (16) and (17) is found to take only negative values. When the number $n$ increases, the number of oscillations increases as well.

In Fig. 1, one can see that $Q(0)=$ const and $Q(a) \rightarrow-1$ at $a \rightarrow \infty$. The local maximum corresponds to the point $a=M$.

The quantum correction $p_{q}$ to the pressure is shown in Fig. 2. This quantum correction $p_{q}$ takes negative

ISSN 2071-0194. Ukr. J. Phys. 2018. Vol. 63, No. 3 
values near the origin $a=0$ and at large values of $a$. It passes through the point $p_{q}=0$ at $a<1$ and then takes positive values. At $a>1$, the pressure fluctuates changing from positive to negative, and vice versa.

The peculiarities of the behavior of the pressure $p_{q}$ manifest themselves in the behavior of the deceleration parameter $q=-\frac{a \ddot{a}}{\dot{a}^{2}}$ drawn in Fig. 3. It is found that when one considers the quantum corrections for the universe filled with dust and radiation, the universe changes from the deceleration (near the initial cosmological singularity at $a=0$ ) to the accelerating expansion for small $a$. The deceleration parameter takes negative and positive values demonstrating that the expansion of the early universe is decelerating, then accelerating, and so on. Extrapolating this solution to the case of larger values of $n$, we come to the conclusion that the quantum corrections generate an additional attraction in the universe acting as dark matter or a repulsion reproducing the effects of dark energy. The quantum corrections can, in principle, induce both the inflationary expansion in the early universe $(n>1)$ and the accelerating expansion in later times $\left(n \sim 10^{120}\right)$.

The way how the quantum corrections exhibit themselves on the total energy density $\rho_{\text {tot }}=\rho_{m}+$ $+\rho_{\gamma}+\rho_{q}$ can be seen in Fig. 4 . The two minima are given by the minima of the quantum correction $\rho_{q}$ shown in Fig. 1. The domains near the minima correspond to the values of the cosmological scale factor, at which the quantum correction exceeds the contribution from the ordinary matter to the total energy density in the very early universe. The bulge of a curve near $a=M$ can be considered as a density fluctuation stipulated by a corresponding growth of the pressure $p_{q}$ from negative values to positive values for $a<M$ and the subsequent change to negative values for $a>M$, shown in Fig. 2. In general case, there are $n-1$ fluctuations for any value of $n$. Such fluctuations may play the role of seeds for the future large-scale structure.

In the domain $a>2 M$, the quantum correction $\rho_{q}$ has the form $\rho_{q}=-\frac{\sigma^{2}}{a^{6}}$, where $\sigma^{2}$ weakly depends on $a$ (see Fig. 1). As was shown in Ref. [7], $\sigma^{2}=$ $=\frac{3}{8} N^{2}$, where $N$ is the number of particles with spin $\frac{1}{2}$ of the Weyssenhoff fluid in the volume $\frac{1}{2} a^{3}$ $[21,22]$. The term with a similar dependence on $a$ arises in the Einstein-Cartan theory [23,24], where $\rho_{q}=-\left\langle s_{\mu \nu} s^{\mu \nu}\right\rangle$, the space-time averaging is per-

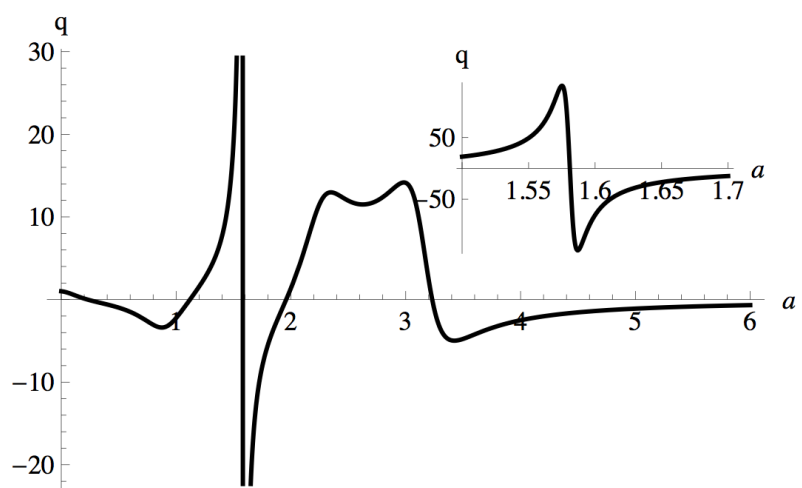

Fig. 3. Deceleration parameter $q$ for the quantum source $Q$ (24) versus the scale factor $a$ for $n=2$

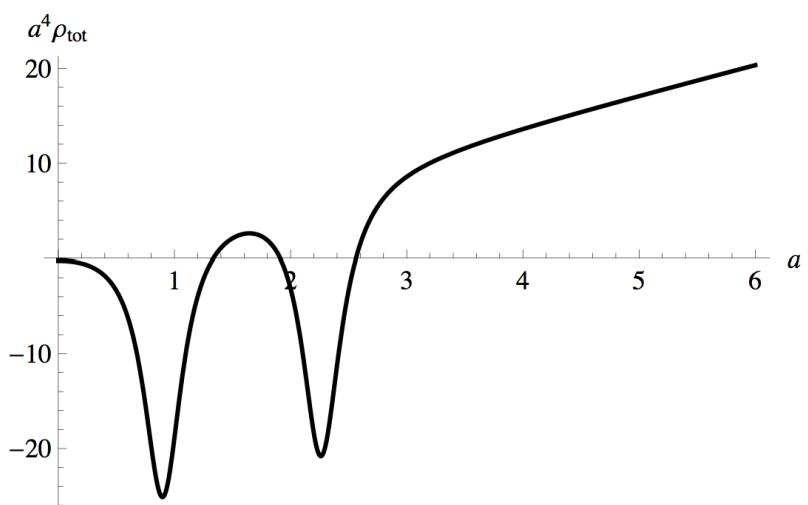

Fig. 4. Total energy density $\rho_{\text {tot }}$ multiplied by $a^{4}$ for the quantum source $Q(24)$ versus the scale factor $a$ for $n=2$

formed in order to make a transition to macroscopic scales, $s_{\mu \nu}$ is the spin density, and it is assumed that the average $\left\langle s_{\mu \nu}\right\rangle=0$.

\section{Conclusion}

The aim of the paper is to demonstrate on a specific example of the exactly solvable model that, in the course of the evolution of the early universe, the cosmological parameters (such as matter-energy density, pressure, and deceleration parameter) can fluctuate under the influence of quantum corrections to gravity. These fluctuations are irregular, forcing the universe to expand with acceleration, then with deceleration, and vice versa. One may say, in the language of classical physics, that the quantum gravity corrections imitate the action of dark energy and dark matter. It appears that the phenomena of inflation in the early universe and the accelerating ex- 
pansion in the current universe, if not entirely, but at least partially, can be explained by the action of quantum component of gravity. These conclusions are made in the framework of non-perturbative quantum theory of gravity based on the quantum generalization of the Hamilton-Jacobi equation. As an example, we have considered the universe filled with dust and radiation. In this case, the non-linear equation for the phase of the wave function admits an analytical solution.

On the Planck scales $(a \sim 1)$, the quantum corrections $\rho_{q}$ can be interpreted as the energy density of matter in the extreme state with the pressure, which fluctuates irregularly relative to the value $p_{q}=0$ (see Fig. 2). As was shown in Ref. [7], with the growth of the scale factor $a$, the quantum corrections behave themselves as a sum of densities,

$\rho_{q} \approx-\frac{\sigma^{2}}{a^{6}}-\frac{1}{a^{4}}$,

where $\sigma^{2} \approx n$ in the domain $a>2 M$. The first term corresponds to the energy density of the Weyssenhoff fluid $[21,22]$, while the second term brings a small negative contribution to the energy density of radiation. Thus, the quantum theory of gravity under consideration in the minisuperspace demonstrates that the quantum corrections to the energy density of matter in the universe describe real matter which, depending on the scale of the variable $a$, takes firstly the form of an extremely strong fluctuating medium, then it turns into the Weyssenhoff fluid, and approaches the behavior of an antigravitating matter with the equation of state $p_{q}=-\frac{1}{3 a^{4}}$ in the final stage.

1. C. Bennett et al. Nine-year wilkinson microwave anisotropy probe (WMAP) observations: Final maps and results. Astrophys. J. Suppl. 208, 20 (2013), arXiv: 1212.5225 [astroph.CO].

2. G. Hinshaw et al. Nine-year wilkinson microwave anisotropy probe (WMAP) observations: Cosmological parameter result. Astrophys. J. Suppl. 208, 19 (2013), arXiv: 1212.5226 [astro-ph.CO].

3. P.A.R. Ade et al. Planck 2013 results. I. Overview of products and scientific results. (Planck Collaboration), arXiv: 1303.5062 [astro-ph.CO].

4. V.E. Kuzmichev, V.V. Kuzmichev. The Big Bang quantum cosmology: The matter-energy production epoch. Acta Phys. Pol. B 39, 979 (2008), arXiv: 0712.0464 [gr-qc].

5. V.E. Kuzmichev, V.V. Kuzmichev. Accelerating quantum universe. Acta Phys. Pol. B 39, 2003 (2008), arXiv: 0712.0465 [gr-qc].
6. V.E. Kuzmichev, V.V. Kuzmichev. Quantum universe on extremely small space-time scales. Ukr. J. Phys. 55, 626 (2010).

7. V.E. Kuzmichev, V.V. Kuzmichev. Quantum corrections to the dynamics of the expanding universe. Acta Phys. Pol. $B$ 44, 2051 (2013), arXiv: 1307.2383 [gr-qc].

8. V.E. Kuzmichev, V.V. Kuzmichev. Quantum geometrodynamical description of the dark sector of the matter-energy content of the universe. Ukr. J. Phys. 60, 664 (2015).

9. B.F. Schutz. Perfect Fluids in General Relativity: Velocity Potentials and a Variational Principle. Phys. Rev. D 2, 2762 (1970).

10. P.A.M. Dirac, Lectures on Quantum Mechanics (Belfer Graduate School of Science, Yeshiva University, 1964).

11. J.W. Moffat. Noncommutative quantum gravity. Phys. Lett. B491, 345 (2000), arXiv: hep-th/0007181.

12. A.H. Chamseddine. Gravity in complex hermitian spacetime. arXiv: hep-th/0610099.

13. P.J.E. Peebles, B Ratra. The cosmological constant and Dark energy. Rev. Mod. Phys. 75, 559 (2003), arXiv:astro$\mathrm{ph} / 0207347$.

14. R.R. Caldwell, R. Dave, P.J. Steinhardt. Cosmological imprint of an energy component with general equation of state. Phys. Rev. Lett. 80, 1582 (1998).

15. J. P. Ostriker, P.J. Steinhardt. The observational case for a low-density Universe with a non-zero cosmological constant. Nature 377, 600 (1995).

16. M.S. Turner, G. Steigman, L. Krauss. Flatness of the universe: Reconciling theoretical prejudices with observational data. Phys. Rev. Lett. 52, 2090 (1984).

17. R.R. Caldwell. A phantom menace? Cosmological consequences of a dark energy component with super-negative equation of state. Phys. Lett. B 545, 23 (2002).

18. R.R. Caldwell, M. Kamionkowski, N.N. Weinberg. Phantom energy and cosmic doomsday. Phys. Rev. Lett. 91, 071301 (2003), arXiv: astro-ph/0302506.

19. V.E. Kuzmichev, V.V. Kuzmichev. Semi-classical universe near initial singularity. Acta Phys. Pol. B 40, 2877 (2009), arXiv: 0905.4142 [gr-qc].

20. J.B. Hartle, S.W. Hawking. Wave function of the Universe. Phys. Rev. D 28, 2960 (1983).

21. J. Weyssenhoff, A. Raabe. Relativistic dynamics of spinfluids and spin-particles. Acta Phys. Pol. 9, 7 (1947).

22. J. Weyssenhoff, in Max-Planck-Festschrift, edited by B. Kockel, W. Macke, A. Papapetrou (Wissenschaften, 1958).

23. F.W. Hehl, P. von der Heyde, G.D. Kerlick, J.M. Nester. General relativity with spin and torsion: Foundations and prospects. Rev. Mod. Phys. 48, 393 (1976).

24. I.S. Nurgaliev, W.N. Ponomariev. The earliest evolutionary stages of the universe and space-time torsion. Phys. Lett. B 130, 378 (1983).

Received 30.10.17

ISSN 2071-0194. Ukr. J. Phys. 2018. Vol. 63, No. 3 
В.Є. Кузъмичов, В.В. Кузъмичов

\section{КВАНТОВА ДИНАМІКА РАННЬОГО ВСЕСВІТУ}

Р е $з$ ю м е

Квантова гравітація може пролити світло на передісторію всесвіту. Квантові поправки до гравітації діють на динаміку розширення всесвіту. Їхній вплив досліджується на прикладі квантової моделі, що має точний розв'язок. Поправки до густини енергії та тиску приводять до виникнення додатко- вого тяжіння (подібно до темної матерії) або відштовхування (подібно до темної енергії) в квантовій системі гравітуючої матерії та випромінювання. Модель пояснює з єдиної точки зору розширення з прискоренням (інфляцію) у ранньому всесвіті (область відносно малих значень квантових чисел) та пізніший перехід від розширення всесвіту з уповільненням до розширення з прискоренням (область дуже великих значень квантових чисел). Обговорюється генерація первинних флуктуацій густини енергії як результат зміни знака квантової поправки до тиску. 